\title{
Molecular Evolution of the Glycosyltransferase 6 Gene Family in Primates
}

\author{
Eliane Evanovich,, ${ }^{1}$ Patricia Jeanne de Souza Mendonça-Mattos, ${ }^{1}$ and Maria Lúcia Harada ${ }^{2}$ \\ ${ }^{1}$ Laboratório de Genética Humana e Médica, Instituto de Ciências Biológicas, Universidade Federal do Pará, Belém, PA, Brazil \\ ${ }^{2}$ Laboratório de Biologia Molecular "Francisco Mauro Salzano", Instituto de Ciências Biológicas, Universidade Federal do Pará, \\ Belém, PA, Brazil
}

Correspondence should be addressed to Eliane Evanovich; lianevanovich@gmail.com

Received 12 July 2016; Accepted 20 October 2016

Academic Editor: Stefano Pascarella

Copyright (c) 2016 Eliane Evanovich et al. This is an open access article distributed under the Creative Commons Attribution License, which permits unrestricted use, distribution, and reproduction in any medium, provided the original work is properly cited.

Glycosyltransferase 6 gene family includes ABO, Ggtal, iGb3S, and GBGT1 genes and by three putative genes restricted to mammals, GT6m6, GTm6, and GT6m7, only the latter is found in primates. GT6 genes may encode functional and nonfunctional proteins. Ggtal and GBGT1 genes, for instance, are pseudogenes in catarrhine primates, while iGb3S gene is only inactive in human, bonobo, and chimpanzee. Even inactivated, these genes tend to be conversed in primates. As some of the GT6 genes are related to the susceptibility or resistance to parasites, we investigated (i) the selective pressure on the GT6 paralogs genes in primates; (ii) the basis of the conservation of iGb3S in human, chimpanzee, and bonobo; and (iii) the functional potential of the GBGT1 and GT6m7 in catarrhines. We observed that the purifying selection is prevalent and these genes have a low diversity, though ABO and Ggtal genes have some sites under positive selection. GT6m7, a putative gene associated with aggressive periodontitis, may have regulatory function, but experimental studies are needed to assess its function. The evolutionary conservation of iGb3S in humans, chimpanzee, and bonobo seems to be the result of proximity to genes with important biological functions.

\section{Introduction}

Glycosyltransferases catalyze the biosynthesis of glycoconjugates and polysaccharides by addition of sugar residues to an acceptor substrate that produce important antigens in the signaling process and recognition by the immune system [1], consisting of more than 90 carbohydrate-active enzymes, grouped by protein similarities in the CAZY database (http://www.cazy.org/GlycosylTransferases.html). Glycosyltransferase 6 are type II transmembrane proteins localized in the Golgi complex. They have a general structure with a cytoplasmic tail, a transmembrane domain, and a large catalytic domain [1-3]. The family includes functional and nonfunctional proteins encoded by ABO, Ggtal, iGb3S, and GBGT1 genes and by three putative genes restricted to mammals: GT6m6, GTm6, and GT6m7; only the latter is found in primates [3].

$\mathrm{ABO}$ gene encodes $\mathrm{A}$ and $\mathrm{B}$ transferases that add $\mathrm{N}$ acetyl-D-galactosamine (GalNAc) or D- galactose (Gal) to
$\mathrm{H}$ substance that produce $\mathrm{A}$ or $\mathrm{B}$ antigens, respectively. $\mathrm{O}$ allele is nonfunctional $[4,5]$. Individuals with the $\mathrm{O}$ phenotype exhibit more resistance to severe malaria caused by Plasmodium falciparum than others with A and/or B types [6-8]. Nonetheless, analysis of this locus in primates showed that the alleles were maintained by balanced selection [9].

Ggtal gene encodes the enzyme $\alpha 1,3$-galactosyltransferase $(\alpha 1,3-\mathrm{GT})$. It transfers UDP-Gal to $\mathrm{N}$-acetyllactosamine and produces $\alpha$-Gal epitope. Catarrhini (human, apes, and Old World monkeys) produce anti-Gal antibody $[10,11]$. According to Galili [12] the inactivation of that gene assured the emergence of this lineage. However, the functionality of Ggtal is maintained in noncatarrhine mammals [13]. The synthesis of $\alpha$-Gal epitopes was also attributed to $i G b 3 S$ gene in mouse. However, this gene encodes isogloboside b3 synthase by adding UDP-Gal to lactosylceramide that produces isogloboside b3 [14].

GBGT1 gene encodes the Forssman synthase (FS) via GalNAc addition to globotriaosylceramide. The result of this 
reaction is Forssman antigen, absent in catarrhine primates due two nonsynonymous substitutions at residues 230 ( $\mathrm{G}$ > S) and $269(\mathrm{Q}>\mathrm{R})[5,15]$.

GT6 member putative genes do not appear to produce an enzyme with catalytic activity due to lack of six motifs LBR-B, LBR-C, LBR-F, LBR-G, LBR-H, and LBR [3].

Casals et al. [16] analyzed the molecular evolution of GBGT1, iGb3S, and GTm7 in two human populations and surprisingly described that these supposed pseudogenes evolve under positive selection. Disagreeing with what would be expected for a nonfunctional sequence, although initially described as pseudogenes, some studies have shown the possibility of GT6m7 being functional. Schaefer et al. [17] found an association between this pseudogene and aggressive periodontitis in three European populations. The same association was strengthened by Hashim et al. [18].

According to Svensson et al. [19], three criteria are important to establish a pseudogene: (1) detrimental mutation (mutations, stop codon, frameshift, splice-site alterations, etc.); (2) number of nonsynonymous substitutions per nonsynonymous site $(\mathrm{Ka} / \mathrm{Ks}$ ratio) that indicate absence of selective pressure; and (3) when the sequences are not overlapping any known gene.

Nevertheless, there are some controversial facts surrounding these criteria: some discoveries have found that some putative pseudogenes participate in DNA, RNA, and protein regulation and that their gene sequences were conserved for millions of years, when they should be evolving neutrally. Therefore, reviewing the pseudogene condition seemed to be important.

Like some of the GT6 genes are related to the susceptibility or resistance to various parasites, their evolutionary and functional analysis is important. Thus, we decided to investigate (i) the selective pressure on the GT6 paralogs genes in primates; (ii) the cause of the conservation of iGb3S pseudogenes in human, chimpanzee, and bonobo; and (iii) the functional potential of the GBGT1 and GT6m7 in catarrhines based on the literature and annotated protein database.

\section{Material and Methods}

2.1. Database and Alignment. The sequences used were retrieved from NCBI (https://www.ncbi.nlm.nih.gov/) and/ or Ensembl databases (http://www.ensembl.org/index. html). The identification of the species used for each database and their accession numbers are presented in Additional file (Table S1 in Supplementary Material available online at http://dx.doi.org/10.1155/2016/9051727).

We analyzed only exons 6 and 7 of the ABO gene which encode the catalytic domain of the protein (comprising 823 of $1062 \mathrm{bp}$, resp.). Exons 1 to 5 are small (their all length is $237 \mathrm{bp}$ ) and they are absent or removed by alternative splicing of transcripts in many different tissues of mammals. The sequences were aligned using the MUSCLE of the MEGA 6 [20]. The best-fit model of evolution for each gene was estimated by Modeltest 3.7 [21] and the phylogenetic tree for
100 replicates was built using PhyML 3.0 [22]. Nucleotide diversity $(\pi)$ was calculated by MEGA 6 [20].

2.2. Detection of Recombination Breakpoints. Recombination was performed by GARD methods available at the http:// www.datamonkey.org/ server [23] under HKY85 model using the Kishino-Hasegawa test $(\mathrm{KH})$. The rate variation was implemented by a general discrete distribution with three rate classes [23].

2.3. Analysis of Molecular Evolution. Molecular evolution was verified by Codeml program implemented in PAML 4 package [24] and by HyPhy in Datamonkey server [23]. Codeml was initially running the M0 model to obtain phylogenetic trees which subsequently were inputted in site models and "free-ratio" branch model $[25,26]$.

Likelihood ratio test (LRT) with $\chi^{2}(\alpha=0.05)$ was used to compare null models with the alternative models (M1a versus $\mathrm{M} 2 \mathrm{a}$ and $\mathrm{M} 7$ versus $\mathrm{M} 8$ ) and M0 versus free-ratio model [25-28] and LRT with critical values of 2.71 at $5 \%$ and 5.41 at $1 \%$ for comparing the nested pair M8a versus M8 test. SLAC, FEL, and FUBAR methods of the Hyphy were used to identify sites under positive or purifying selection. Evidence of episodic positive selection was verified by MEME (applied to individual sites) and BUSTED (applied to gene-wide with primates as foreground branch) [29].

These methods use different confidence index as $p$ value, Bayes factor, and posterior probability. $p$ value was significant if it is less than or equal to 0.05 (BUSTED, SLAC, FEL, and MEME models), Bayer factor $\geq 50$ (REL), and posterior probability $\geq 95 \%$ (FUBAR). The models of evolution used in these analyses were REV (for ABO, Ggtal, and GT6m7) and HKY85 (for GBGT1 and $i G b 3 S$ ).

2.4. Annotated Protein Database. To obtain more functional information, tissue expression, and protein interactions on each of the genes, we searched databases neXtProt (https://www.nextprot.org/), UniProtKB (http:// www.uniprot.org/), GeneCards (http://www.genecards.org/), and String (http://string-db.org/).

\section{Results and Discussions}

GARD method found evidence of recombination only in the GT6m7 sequence at position 190. PARRIS algorithm indicates that the genes are strong purifying selection, and BUSTED does not find evidence of diversifying selection under each whole gene. However, some sites were identified by the MEME as under episodic selection pressures (Table 1). The results obtained for each of the genes are shown in Table 1.

3.1. ABO. ABO analyses performed by Codeml models inferred the site 266 under positive selection. This codon position is crucial for differentiating $\mathrm{A}$ and $\mathrm{B}$ alleles in primates $[9,30]$. Although the M8 is not concise as the M2a model [23], the presence of diversifying and positive selection was indicated by MEME and "free-ratio" model. MEME algorithm suggested diversifying selection on site 
TABLE 1: Codons under positive selection or diversifying selection with values significant obtained by Codeml or HyPhy methods.

\begin{tabular}{lcc}
\hline Gene & Codon position & Method \\
\hline \multirow{2}{*}{ ABO } & 266 & M7-M8 \\
& 268 & MEME \\
Ggtal & 109 & M7-M8 \\
& 250 & MEME, SLAC \\
\multirow{2}{*}{ iGb3S } & 361 & FEL, MEME \\
GBGT1 & 96 & FEL, MEME \\
GT6m7 & $135,228,233$ & MEME \\
& $12,237,266$ & MEME \\
& 96 & FEL, MEME \\
\hline
\end{tabular}

268 ( $p$ value of 0.002 ). "Free-ratio" test showed that some branches evolve on positive selection. The results obtained corroborate to balancing selection described by Ségurel et al. [9]. According to some authors, certain alleles may act against pathogens and are maintained during the evolution [31-34]. In human populations, individuals with A or B types seem to be less susceptible to infections caused by Helicobacter pylori and Vibrio cholerae compared to the carriers of $\mathrm{O}$ allele [32, 35]; in contrast, greater resistance to severe malaria caused by Plasmodium falciparum in blood group $\mathrm{O}$ individuals due to reduced rosette formation in erythrocytes was observed $[6,36]$. Results are shown in additional file: Tables S2-S4 and Figure S1.

3.2. Ggtal. None of the sites found by algorithms are involved in the protein activity in accordance with the inferred structure by Gastinel et al. [37] for bovine 1,3-Gal. Positive selection was indicated by comparison of M7 versus M8 models $(\mathrm{LTR}=6.367 ; p<0.05)$ in position $109(\mathrm{E}>\mathrm{K})$; however, it substitution is restricted to Gttal pseudogene of Angola colobus, thus expected.

250 and 351 site codons were found on pervasive diversifying selection by MEME and, respectively, by SLAC and FEL. Codon 250 is localized in $\alpha 1,3-$ GalT catalytic pocket. It is involved in the stability of the C-terminal segment [37] and is a binding site for disaccharide acceptor substrates [38]. The squirrel monkey $\alpha 1,3-$ GalT presents substitution of tryptophan to phenylalanine in this residue, but this change does not seem to have a large impact on the catalytic activity of the enzyme [38]. Codon 351 is near to C-terminal portion and in this analysis it presents very polymorphism, having polar and nonpolar amino acids in different mammals, but they do not seem to result in major changes in protein structure.

Results of "free-ratio" test indicate different selective pressures between the several lineages, especially in howler that shows the highest $\omega$ values (0.64), similar to results found by Koike et al. [13] (additional file: Tables S5-S7 and Figure S2).

3.3. $i G b 3 S$. Only the site 97 was pointed out as being under positive selection by FEL. It was also indicated by the MEME, such as sites 136, 229, and 275. Among them, 97, 229, and 275 positions are most relevant information, because amino acids are preserved in different taxa showing a strong selective pressure on protein in mammals (Figure 1) [39]. Substitutions in these residues are not involved in important enzymatic processes. However, the polarity changes involving residue 275 as observed in iGb3S protein of the Old World monkeys (G275R) may result in some change in conformational entropy of the backbone in the local coil of the protein (see [40]), because this residue is in a hydrophobic site on $\alpha$-helix $(\alpha 5)$.

This gene is inactive in humans due to multiple mutations, including Y252N and L187P substitutions, but the high conservation of the putative pseudogene in human populations (nucleotide diversity, $\pi=0.0010$ ) was reported by Casals et al. [16].

$i G b 3 S$ has no evidence of recombination and it is syntenically conserved in mammals. Moreover, it is flanked by two conserved genes (ZNF362, Zinc finger protein 362 and PHC2, polyhomeotic homolog 2 genes), suggesting that their conservation in humans may result from their chromosomic position that is under strong selective pressure (additional file: Tables S8-S10).

3.4. GBGT1. PARRIS indicates $82 \%$ sites under purifying selection $(\omega$ mean $=0.270)$ and particularly in Catarrhini that present low diversity $(\pi=0.030)$. (Results are shown in additional file: Table S11-S13.) These primates are Forssmannegative, instead of Forssman glycolipid. Some cells of these primates show the precursor glycolipids globotriaosylceramide and globoside that are used as a binding site for bacteria, viruses, and toxins [15, 41-44]. This is apparently an adaptive disadvantage, but it can be offset.

For instance, individuals whose expression is Forssman antigen ( $\mathrm{ABO}$ subgroup $\mathrm{A}_{\mathrm{pae}}$ ) [19] present protection against Shiga toxin 1 (Stxl) and vulnerability to Stx2a toxin, variant of Stx2 though [45, 46]. Louise and Obrig [47] analyze patients with hemolytic uremic syndrome (HUS) caused by Escherichia coli O157: H7 and observed that Stx2a toxin is 1000 times more cytotoxic than Stxl. Other epidemiological data using baboon and mouse models also noticed it [47-49]. It is possible that it is an evolutionary advantage, presenting a key role in the evolution of Catarrhini.

3.5. GT6m7. Our results indicated high conservation of nucleotides and purifying selection ( $\omega$ mean of 0.475 ) as found by Casals et al. [16], although positive selection was detected at codon 126 by SLAC, FEL, MEME, and FUBAR (additional file: Tables S14-S16).

There is missing information on the protein structure, although its higher expression in the testis, leukocytes, and gingiva [17] is quite instigating. The most relevant information obtained was related to its regulatory potential, which is explained below.

In a genome-wide association study (GWAS), Schaefer et al. [17] found relation between an allele of SNP rs1537415 (localized at intron 2 of $G T 6 m 7$ ) to aggressive periodontitis $(\mathrm{AgP})$ in three European populations. More recently, this 


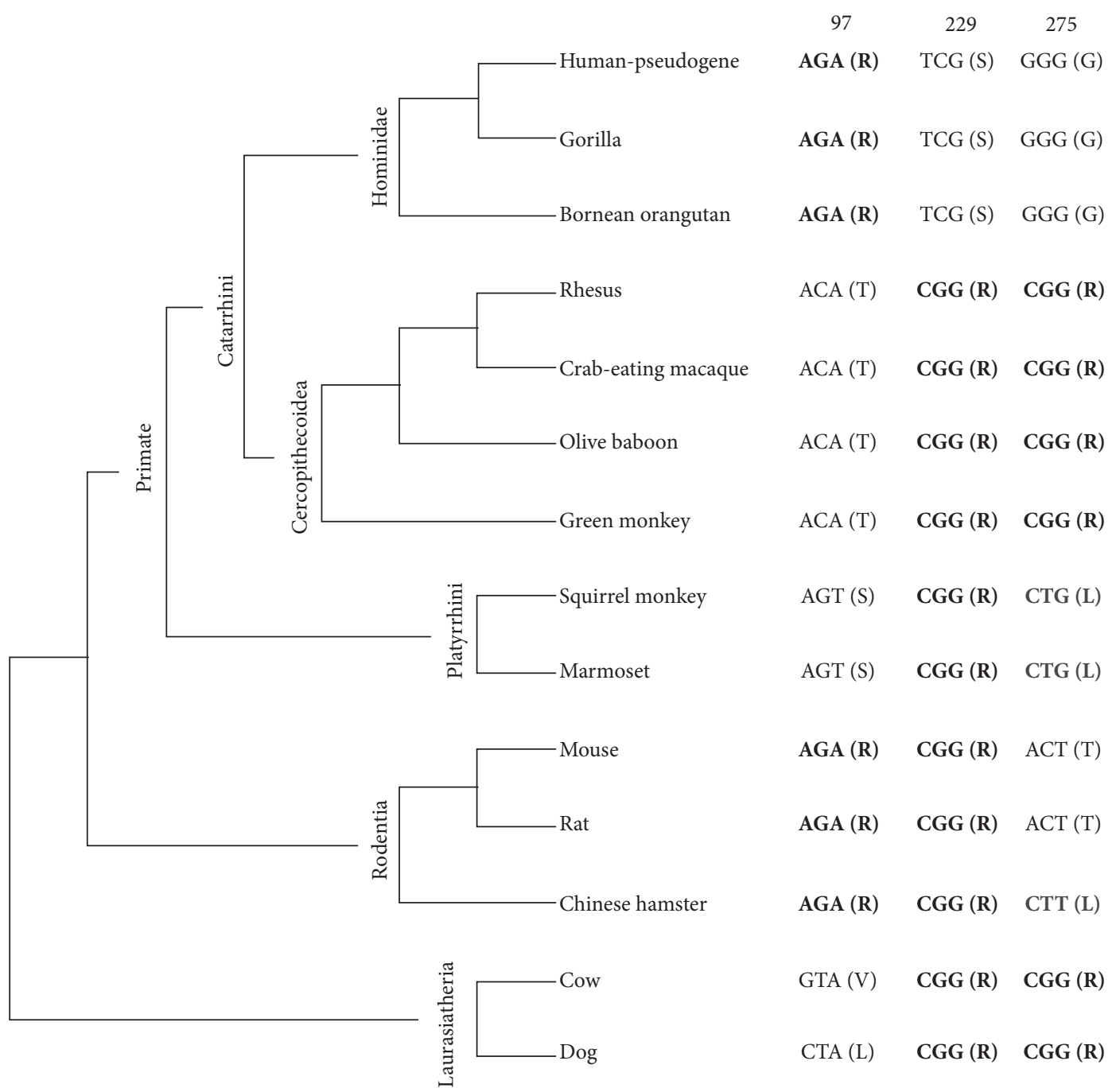

FIgure 1: Phylogenetic tree for $i G b 3$ gene indicated three sites under diversifying selection in accordance with MEME. The sites that are shared by different taxa are highlighted in bold. Tree constructed using PhyML, 100 replicates, under GTR + G model. $L n l=-4263.35$. Values of branches sizes and bootstrap were omitted.

association was found in Sudanese population [18]. Schaefer et al. [17] using computational analysis reported that substitution from C to G in SNP rs1537415 may decrease the affinity between the transcriptional factor GATA-3 and its binding site.

We submitted intron 2 to BLASTN Ensembl against Human GRCh38 and we found $a$ fragment of $353 \mathrm{bp}$ (121 bp upstream to SNP rs1537415) with greater identity than $90 \%$ to the following regulatory sequences: ADGRL3-AS1 (adhesion G protein-coupled receptor L3 antisense RNA), RUNX1 (runt-related transcription factor 1), SRBD1 (S1 RNA binding domain 1 gene), CTND2 (catenin delta 2), and FEZ2 (Fasciculation and Elongation Protein Zeta 2). The values obtained for the five best hits are in additional file: Figure S3.

In recent decades, the function of some mammalian pseudogenes was clarified, acting at different levels, from DNA to protein [50-55]. For instance, the transcription of the Makorin1 gene is regulated by its pseudogene paralogous Makarin1-p1 [55]; and HMGA1 (high mobility group A1) presents regulatory action on the insulin receptor gene (INSR) [55]. The data presented suggest that GT6m7 also has some functionality, but experimental data are needed.

\section{Conclusion}

As shown in this paper, the purifying selection is prevalent in primate GT6 genes and has a low diversity ( $\omega$ mean $=0.342$ and $\pi$ mean $=0.0822$ ). Surprisingly, their retention was found even in taxa where one of the genes is inactivated. ABO, Ggtal, and GBGT1 genes appear to be under strong selective pressures exerted by pathogens. $\mathrm{ABO}$ locus evolved in a balanced manner in primates with the maintenance of the three major alleles in at least ten species, including human, orangutan, and squirrel monkey. Ggtal gene also gives advantage against pathogens with expression of $\alpha$-Gal epitopes or anti-Gal antibody [12]. Similarly, the absence of expression of Forssman antigen is relevant against 
bacterial infections. However, GT6m7 and $i G b 3 S$ seem to evolve differently. GT6m7, in primates, may have regulatory function. Otherwise, the maintenance of $i G b 3 S$ in humans, chimpanzee, and bonobo seems to be the only result of the proximity to genes with important biological functions.

\section{Disclosure}

Part of this work was presented in Eliane Evanovich's Master thesis [56].

\section{Competing Interests}

The authors have declared that no competing interests exist.

\section{Acknowledgments}

The work was supported by Coordenação de Aper-feiçoamento de Pessoal de Nível Superior (CAPES) from the Education Ministry, Brazil. Part of this work was presented in Eliane Evanovich's Master thesis [56].

\section{References}

[1] A. Varki, "Biological roles of oligosaccharides: all of the theories are correct," Glycobiology, vol. 3, no. 2, pp. 97-130, 1993.

[2] J. C. Paulson and K. J. Colley, "Glycosyltransferases. Structure, localization, and control of cell type-specific glycosylation," Journal of Biological Chemistry, vol. 264, no. 30, pp. 17615-17618, 1989.

[3] A. L. Turcot-Dubois, B. Le Moullac-Vaidye, S. Despiau et al., "Long-term evolution of the CAZY glycosyltransferase 6 (ABO) gene family from fishes to mammals - a birth-and-death evolution model," Glycobiology, vol. 17, no. 5, pp. 516-528, 2007.

[4] F. Yamamoto, "Review: ABO blood group system-ABH oligosaccharide antigens, anti-A and anti- $\mathrm{B}, \mathrm{A}$ and $\mathrm{B}$ glycosyltransferases and ABO genes," Immunohematology, vol. 20, no. 1, pp. 3-22, 2004.

[5] F. Yamamoto, E. Cid, M. Yamamoto, and A. Blancher, "ABO research in the modern era of genomics," Transfusion Medicine Reviews, vol. 26, no. 2, pp. 103-118, 2012.

[6] A. E. Fry, M. J. Griffiths, S. Auburn et al., "Common variation in the $\mathrm{ABO}$ glycosyltransferase is associated with susceptibility to severe Plasmodium falciparum malaria," Human Molecular Genetics, vol. 17, no. 4, pp. 567-576, 2008.

[7] A. K. Panda, S. K. Panda, A. N. Sahu, R. Tripathy, B. Ravindran, and B. K. Das, "Association of ABO blood group with severe falciparum malaria in adults: case control study and metaanalysis," Malaria Journal, vol. 10, article no. 309, 2011.

[8] M. J. Mackinnon, C. Ndila, S. Uyoga et al., "Environmental correlation analysis for genes associated with protection against malaria," Molecular Biology and Evolution, vol. 33, no. 5, pp. 1188-1204, 2016.

[9] L. Ségurel, E. E. Thompson, T. Flutre et al., "The ABO blood group is a trans-species polymorphism in primates," Proceedings of the National Academy of Sciences of the United States of America, vol. 109, no. 45, pp. 18493-18498, 2012.

[10] W. M. Blanken and D. H. Van den Eijnden, "Biosynthesis of terminal Gal $\alpha 1 \rightarrow 3 \mathrm{Gal} \beta 1 \rightarrow 4 \mathrm{GlcNAc}-\mathrm{R}$ oligosaccharide sequences on glycoconjugates. Purification and acceptor specificity of a UDP-Gal:N-acetyllactosaminide $\alpha 1$,3galactosyltransferase from calf thymus," The Journal of Biological Chemistry, vol. 260, pp. 12927-12934, 1985.

[11] G. Huai, P. Qi, H. Yang, and Y. Wang, "Characteristics of $\alpha$ Gal epitope, anti-Gal antibody, $\alpha 1,3$ galactosyltransferase and its clinical exploitation (Review)," International Journal of Molecular Medicine, vol. 37, no. 1, pp. 11-20, 2016.

[12] U. Galili, "Significance of the Evolutionary $\alpha 1,3$-galactosyltransferase (GGTA1) gene inactivation in preventing extinction of apes and old world monkeys," Journal of Molecular Evolution, vol. 80, no. 1, pp. 1-9, 2015.

[13] C. Koike, M. Uddin, D. E. Wildman et al., "Functionally important glycosyltransferase gain and loss during catarrhine primate emergence," Proceedings of the National Academy of Sciences of the United States of America, vol. 104, no. 2, pp. 559-564, 2007.

[14] J. Milland, D. Christiansen, B. D. Lazarus, S. G. Taylor, P. X. Xing, and M. S. Sandrin, "The molecular basis for $\mathrm{Gal} \alpha(1,3) \mathrm{Gal}$ expression in animals with a deletion of the $\alpha 1,3$ galactosyltransferase gene," The Journal of Immunology, vol. 176, no. 4, pp. 2448-2454, 2006.

[15] H. Xu, T. Storch, M. Yu, S. P. Elliott, and D. B. Haslam, "Characterization of the human Forssman synthetase gene. An evolving association between glycolipid synthesis and hostmicrobial interactions," The Journal of Biological Chemistry, vol. 274, no. 41, pp. 29390-29398, 1999.

[16] F. Casals, A. Ferrer-Admetlla, M. Sikora et al., "Human pseudogenes of the $\mathrm{ABO}$ family show a complex evolutionary dynamics and loss of function," Glycobiology, vol. 19, no. 6, pp. 583-591, 2009.

[17] A. S. Schaefer, G. M. Richter, M. Nothnagel et al., "A genomewide association study identifies GLT6D1 as a susceptibility locus for periodontitis," Human Molecular Genetics, vol. 19, no. 3, pp. 553-562, 2009.

[18] N. T. Hashim, G. J. Linden, M. E. Ibrahim et al., "Replication of the association of GLT6D1 with aggressive periodontitis in a Sudanese population," Journal of Clinical Periodontology, vol. 42, no. 4, pp. 319-324, 2015.

[19] Ö. Svensson, L. Arvestad, and J. Lagergren, "Genome-wide survey for biologically functional pseudogenes," PLoS Computational Biology, vol. 2, no. 5, p. e46, 2006.

[20] K. Tamura, G. Stecher, D. Peterson, A. Filipski, and S. Kumar, "MEGA6: molecular evolutionary genetics analysis version 6.0," Molecular Biology and Evolution, vol. 30, no. 12, pp. 2725-2729, 2013.

[21] D. Posada and K. A. Crandall, "MODELTEST: testing the model of DNA substitution," Bioinformatics, vol. 14, no. 9, pp. 817-818, 1998.

[22] S. Guindon, J.-F. Dufayard, V. Lefort, M. Anisimova, W. Hordijk, and O. Gascuel, "New algorithms and methods to estimate maximum-likelihood phylogenies: assessing the performance of PhyML 3.0," Systematic Biology, vol. 59, no. 3, pp. 307-321, 2010.

[23] S. L. K. Pond, S. D. W. Frost, and S. V. Muse, "HyPhy: hypothesis testing using phylogenies," Bioinformatics, vol. 21, no. 5, pp. 676679, 2005.

[24] Z. Yang, "PAML 4: phylogenetic analysis by maximum likelihood," Molecular Biology and Evolution, vol. 24, no. 8, pp. 15861591, 2007.

[25] Z. Yang and R. Nielsen, "Synonymous and nonsynonymous rate variation in nuclear genes of mammals," Journal of Molecular Evolution, vol. 46, no. 4, pp. 409-418, 1998. 
[26] Z. Yang, R. Nielsen, N. Goldman, and A.-M. K. Pedersen, "Codon-substitution models for heterogeneous selection pressure at amino acid sites," Genetics, vol. 155, no. 1, pp. 431-449, 2000.

[27] W. J. Swanson, R. Nielsen, and Q. Yang, "Pervasive adaptive evolution in mammalian fertilization proteins," Molecular Biology and Evolution, vol. 20, no. 1, pp. 18-20, 2003.

[28] W. S. W. Wong, Z. Yang, N. Goldman, and R. Nielsen, "Accuracy and power of statistical methods for detecting adaptive evolution in protein coding sequences and for identifying positively selected sites," Genetics, vol. 168, no. 2, pp. 1041-1051, 2004.

[29] B. Murrell, S. Weaver, M. D. Smith et al., "Gene-wide identification of episodic selection," Molecular Biology and Evolution, vol. 32, no. 5, pp. 1365-1371, 2015.

[30] E. Evanovich and M. L. Harada, "Primate ABO gene is under weak positive selection," Notulae Scientia Biologicae, vol. 4, pp. 7-12, 2012.

[31] N. Otting, N. G. De Groot, G. G. M. Doxiadis, and R. E. Bontrop, "Extensive Mhc-DQB variation in humans and non-human primate species," Immunogenetics, vol. 54, no. 4, pp. 230-239, 2002.

[32] D. J. Anstee, “The relationship between blood groups and disease," Blood, vol. 115, no. 23, pp. 4635-4643, 2010.

[33] E. M. Leffler, Z. Gao, S. Pfeifer et al., "Multiple instances of ancient balancing selection shared between humans and chimpanzees," Science, vol. 340, no. 6127, pp. 1578-1582, 2013.

[34] L. Azevedo, C. Serrano, A. Amorim, and D. N. Cooper, "Transspecies polymorphism in humans and the great apes is generally maintained by balancing selection that modulates the host immune response," Human Genomics, vol. 9, article 21, 2015.

[35] J. B. Harris, A. I. Khan, R. C. LaRocque et al., "Blood group, immunity, and risk of infection with Vibrio cholerae in an area of endemicity," Infection and Immunity, vol. 73, no. 11, pp. 74227427, 2005.

[36] T. N. Williams, "Human red blood cell polymorphisms and malaria," Current Opinion in Microbiology, vol. 9, no. 4, pp. 388394, 2006.

[37] L. N. Gastinel, C. Bignon, A. K. Misra, O. Hindsgaul, J. H. Shaper, and D. H. Joziasse, "Bovine $\alpha 1$,3-galactosyltransferase catalytic domain structure and its relationship with $\mathrm{ABO}$ histoblood group and glycosphingolipid glycosyltransferases," The EMBO Journal, vol. 20, no. 4, pp. 638-649, 2001.

[38] Y. Zhang, A. Deshpande, Z. Xie, R. Natesh, K. R. Acharya, and K. Brew, "Roles of active site tryptophans in substrate binding and catalysis by $\alpha-1,3$ galactosyltransferase," Glycobiology, vol. 14, no. 12, pp. 1295-1302, 2004.

[39] D. Christiansen, J. Milland, E. Mouhtouris et al., "Humans lack iGb3 due to the absence of functional iGb3-synthase: implications for NKT cell development and transplantation," PLoS Biology, vol. 6, no. 7, article e172, 2008.

[40] C. N. Pace and J. M. Scholtz, "A helix propensity scale based on experimental studies of peptides and proteins," Biophysical Journal, vol. 75, no. 1, pp. 422-427, 1998.

[41] D. B. Haslam and J. U. Baenziger, "Expression cloning of Forssman glycolipid synthetase: a novel member of the histo-blood group ABO gene family," Proceedings of the National Academy of Sciences of the United States of America, vol. 93, no. 20, pp. 10697-10702, 1996.

[42] G. T. Keusch, M. Jacewicz, M. Mobassaleh, and A. DonohueRolfe, "Shiga toxin: intestinal cell receptors and pathophysiology of enterotoxic effects," Reviews of Infectious Diseases, vol. 13, supplement 4, pp. S304-S310, 1991.
[43] M. Ezzelarab, D. Ayares, and D. K. C. Cooper, "Carbohydrates in xenotransplantation," Immunology and Cell Biology, vol. 83, no. 4, pp. 396-404, 2005.

[44] G. C. Hansson, K.-A. Karlsson, G. Larson, N. Strömberg, and J. Thurin, "Carbohydrate-specific adhesion of bacteria to thinlayer chromatograms: a rationalized approach to the study of host cell glycolipid receptors," Analytical Biochemistry, vol. 146, no. 1, pp. 158-163, 1985.

[45] C. A. Lingwood, "Role of verotoxin receptors in pathogenesis," Trends in Microbiology, vol. 4, no. 4, pp. 147-153, 1996.

[46] S. P. Elliott, M. Yu, H. Xu, and D. B. Haslam, "Forssman synthetase expression results in diminished Shiga toxin susceptibility: a role for glycolipids in determining host-microbe interactions," Infection and Immunity, vol. 71, no. 11, pp. 65436552, 2003.

[47] C. B. Louise and T. G. Obrig, "Specific interaction of Escherichia coli O157:H7-derived Shiga-like toxin II with human renal endothelial cells," Journal of Infectious Diseases, vol. 172, no. 5, pp. 1397-1401, 1995.

[48] V. L. Tesh, J. A. Burris, J. W. Owens et al., "Comparison of the relative toxicities of Shiga-like toxins type I and type II for mice," Infection and Immunity, vol. 61, no. 8, pp. 3392-3402, 1993.

[49] R. L. Siegler, T. G. Obrig, T. J. Pysher, V. L. Tesh, N. D. Denkers, and F. B. Taylor, "Response to Shiga toxin 1 and 2 in a baboon model of hemolytic uremic syndrome," Pediatric Nephrology, vol. 18, no. 2, pp. 92-96, 2003.

[50] S. N. Hirotsune, N. Yoshida, A. Chen et al., "An expressed pseudogene regulates the messenger-RNA stability of its homologous coding gene," Nature, vol. 423, no. 6935, pp. 91-96, 2003.

[51] E. S. Balakirev and F. J. Ayala, "Pseudogenes: are they 'Junk' or functional DNA?" Annual Review of Genetics, vol. 37, pp. 123151, 2003.

[52] T. C. Roberts and K. V. Morris, "Not so pseudo anymore: pseudogenes as therapeutic targets," Pharmacogenomics, vol. 14, no. 16, pp. 2023-2034, 2013.

[53] L. Korrodi-Gregório, J. Abrantes, T. Muller et al., "Not so pseudo: the evolutionary history of protein phosphatase 1 regulatory subunit 2 and related pseudogenes," BMC Evolutionary Biology, vol. 13, no. 1, article no. 242, 2013.

[54] O. Podlaha and J. Zhang, "Nonneutral evolution of the transcribed pseudogene Makorin1-p1 in mice," Molecular Biology and Evolution, vol. 21, no. 12, pp. 2202-2209, 2004.

[55] E. Chiefari, S. Iiritano, F. Paonessa et al., "Pseudogene-mediated posttranscriptional silencing of HMGA1 can result in insulin resistance and type 2 diabetes," Nature Communications, vol. 1, article 40, 2010.

[56] E. B. dos Santos, Evolução molecular do sistema ABO em primatas do novo mundo (Master in Biological Sciences) [M.S. thesis], Universidade Federal do Pará, Centro de Ciências Biológicas, Departamento de Genética, Belém, Brazil, 2001. 

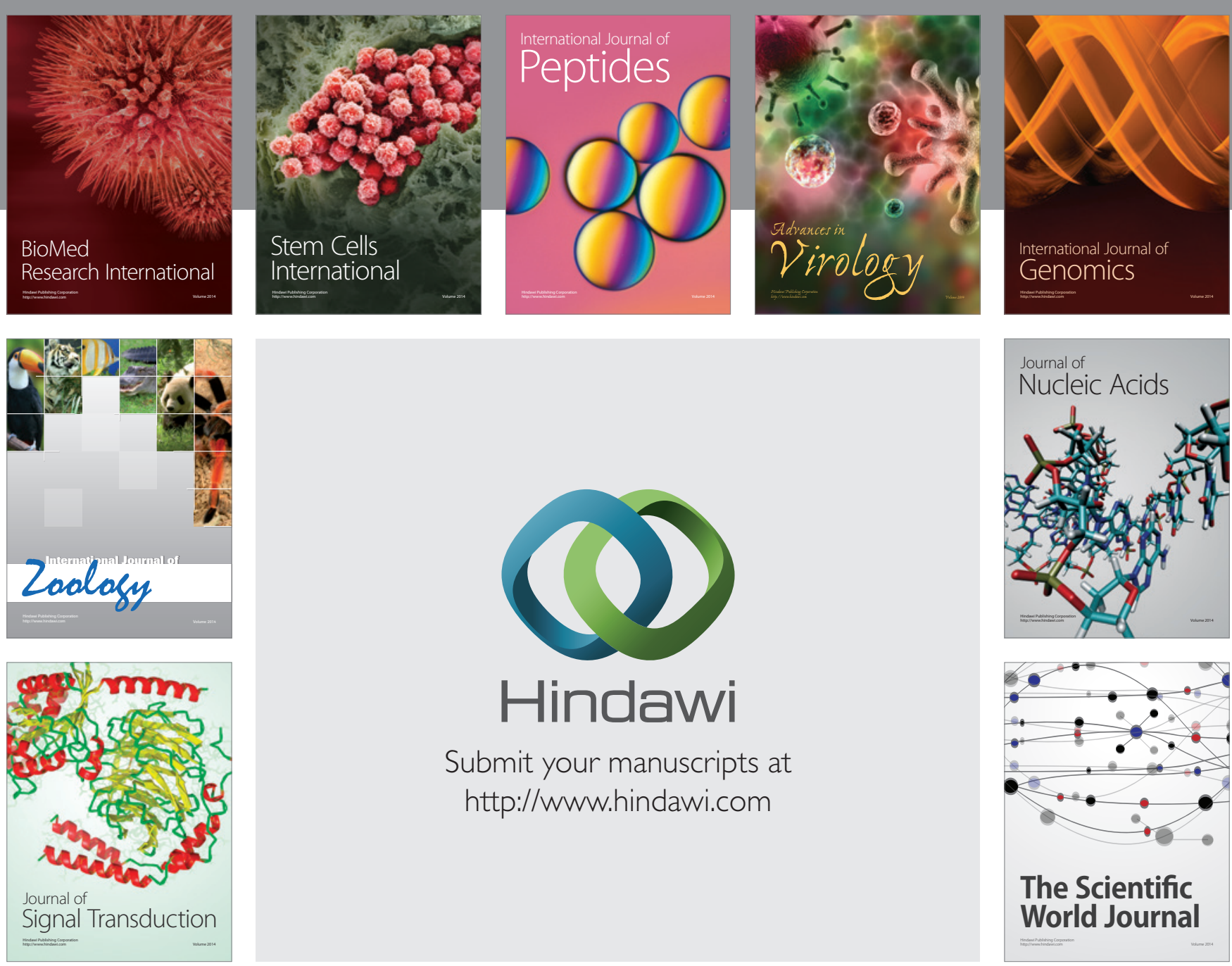

Submit your manuscripts at

http://www.hindawi.com
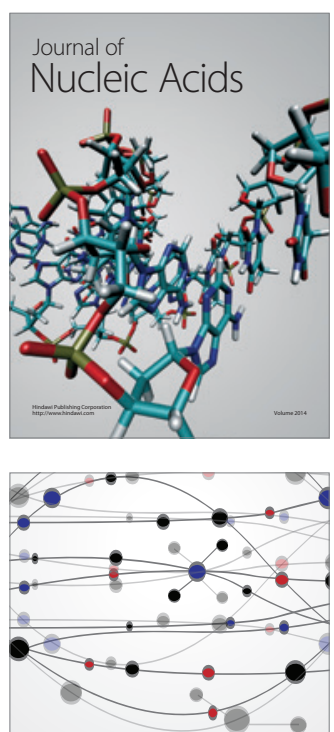

The Scientific World Journal
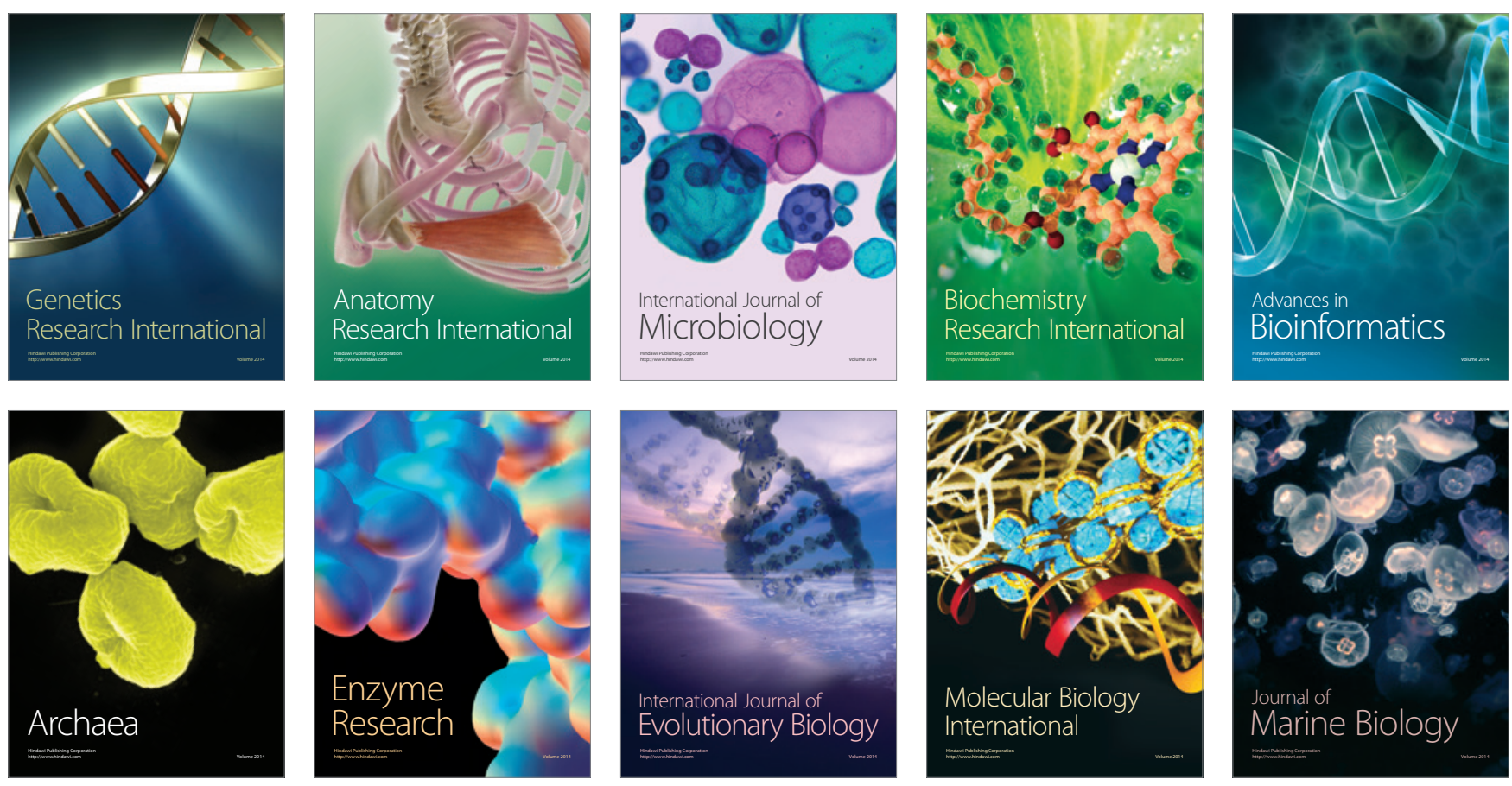